\title{
The Price of Connectivity for Vertex Cover
}

\section{Eglantine Camby ${ }^{1} \quad$ Jean Cardinal $^{1} \quad$ Samuel Fiorini $^{2} \quad$ Oliver Schaudt $\left.^{3}\right|^{k}$}

\author{
${ }^{1}$ Université Libre de Bruxelles (ULB), Département de Mathématique, Belgium \\ ${ }^{2}$ Université Libre de Bruxelles (ULB), Département d'Informatique, Belgium \\ ${ }^{3}$ Université Pierre et Marie Curie, Combinatoire et Optimisation, Paris, France
}

received $7^{\text {th }}$ May 2013, revised $28^{\text {th }}$ Sep. 2013, $4^{\text {th }}$ Mar. 2014, accepted $17^{\text {th }}$ Mar. 2014.

The vertex cover number of a graph is the minimum number of vertices that are needed to cover all edges. When those vertices are further required to induce a connected subgraph, the corresponding number is called the connected vertex cover number, and is always greater or equal to the vertex cover number.

Connected vertex covers are found in many applications, and the relationship between those two graph invariants is therefore a natural question to investigate. For that purpose, we introduce the Price of Connectivity, defined as the ratio between the two vertex cover numbers. We prove that the price of connectivity is at most 2 for arbitrary graphs. We further consider graph classes in which the price of connectivity of every induced subgraph is bounded by some real number $t$. We obtain forbidden induced subgraph characterizations for every real value $t \leq 3 / 2$.

We also investigate critical graphs for this property, namely, graphs whose price of connectivity is strictly greater than that of any proper induced subgraph. Those are the only graphs that can appear in a forbidden subgraph characterization for the hereditary property of having a price of connectivity at most $t$. In particular, we completely characterize the critical graphs that are also chordal.

Finally, we also consider the question of computing the price of connectivity of a given graph. Unsurprisingly, the decision version of this question is NP-hard. In fact, we show that it is even complete for the class $\Theta_{2}^{P}=P^{N P[\log ]}$, the class of decision problems that can be solved in polynomial time, provided we can make $O(\log n)$ queries to an NP-oracle. This paves the way for a thorough investigation of the complexity of problems involving ratios of graph invariants.

Keywords: vertex cover, connected vertex cover, computational complexity, forbidden induced subgraphs

\section{Introduction}

A vertex cover of a graph $G$ is a vertex subset $C$ such that every edge of $G$ has at least one endpoint in $C$. The size of a minimum vertex cover of $G$, denoted by $\tau(G)$, is called the vertex cover number of $G$. The problem of finding a minimum vertex cover in a graph is one of the 21 NP-hard problems identified by Karp in 1972, and has since been intensively studied in the literature.

\footnotetext{
*Parts of this research have been carried out during the visit of Oliver Schaudt to Université Libre de Bruxelles. Emails: \{ecamby, sfiorini, jcardin\}@ulb.ac.be, schaudt@math.jussieu.fr
} 
A well-known variant of the notion of vertex cover is that of connected vertex cover, defined as a vertex cover $C_{c}$ such that the induced subgraph $G\left[C_{c}\right]$ is connected. (If $G$ is not connected we ask that $G\left[C_{c}\right]$ has the same number of component as $G$.) The minimum size of such a set, denoted by $\tau_{c}(G)$, is the connected vertex cover number of $G$. A connected vertex cover of size $\tau_{c}(G)$ is called a minimum connected vertex cover. The connected vertex cover problem has been widely studied in the literature. According to Guo, Niedermeier and Wernicke [6], the question $\tau_{c}(G) \leq k$ can be solved by a fixed-parameter tractable (FPT) algorithm in $O\left(6^{k} n+4^{k} n^{2}+2^{k} n^{2} \log n+2^{k} n m\right)$. Later, Mölle, Richter and Rossmanith [9] found an improved FPT algorithm in $O\left(3.2361^{k}\right)$ whereas Fernau and Manlove [5] designed an $O\left(2.9316^{k}\right)$ algorithm. Finally, Mölle, Richter and Rossmanith [10] refined the original enumerate-and-expand algorithm to obtain a running time of $O\left(2.7606^{k}\right)$. Moreover, Fernau and Manlove [5] showed that the connected vertex cover problem is not approximable within an asymptotic performance ratio of $10 \sqrt{5}-21-\delta$, for any $\delta>0$, unless $P=N P$. Furthermore, Escoffier, Gourvès and Monnot [4] proved that this problem is polynomial in chordal graphs, has a PTAS in planar graphs, is 5/3-approximable in any class of graphs where the vertex cover problem is polynomial, in particular in bipartite graphs.

Our contribution is to study the interdependence of $\tau$ and $\tau_{c}$, both from a complexity-theoretic point of view and in some hereditary classes of graphs.

Let us first note that every vertex cover $C$ of a connected graph $G$ such that $G[C]$ has $c$ connected components can be turned into a connected vertex cover of $G$ by adding at most $c-1$ vertices. This directly yields the following observation.

Observation 1 For every graph $G$ it holds that $\tau_{c}(G) \leqslant 2 \tau(G)-1$.

As an immediate consequence of Observation 11, the following holds for every graph $G$ (with at least one edge):

$$
1 \leqslant \tau_{c}(G) / \tau(G)<2
$$

We define the Price of Connectivity (PoC) of a graph $G$ as the ratio $\tau_{c}(G) / \tau(G)$. Hence we just showed that the Price of Connectivity of any graph lies in the interval $[1,2)$. We denote by $P_{k}$ the path on $k$ vertices and by $C_{k}$ the cycle on $k$ vertices. Note that the upper bound in (1) is asymptotically sharp in the class of paths and in the class of cycles, in the sense that

$$
\lim _{k \rightarrow \infty} \tau_{c}\left(P_{k}\right) / \tau\left(P_{k}\right)=2=\lim _{k \rightarrow \infty} \tau_{c}\left(C_{k}\right) / \tau\left(C_{k}\right) .
$$

Our contribution is split into two parts. In the first part, we consider the computational complexity of the problem of deciding whether the $\mathrm{PoC}$ of a graph given as input is bounded by some constant $t$. We show the completeness of this problem with respect to a well-defined complexity class in the polynomial hierarchy. In the second part, we investigate graph classes in which the PoC of every induced subgraph is bounded by a constant $t$ with $t \in[1,2)$. Those classes will be defined by forbidden induced subgraphs. The forbidden subgraph characterizations directly yields polynomial-time algorithms for recognizing graphs in those classes.

We use the following standard notation. If $G$ and $H$ are two graphs we say that $G$ contains $H$ if $G$ has an induced subgraph isomorphic to $H$. We say that $G$ is $H$-free if $G$ does not contain $H$. Furthermore, we say that $G$ is $\left(H_{1}, \ldots, H_{\ell}\right)$-free if $G$ does not contain $H_{i}$ for any $i \in\{1, \ldots, \ell\}$.

The Price of Connectivity (as defined here) has been introduced by Cardinal and Levy [3, 8], who showed that it was bounded by $2 /(1+\varepsilon)$ in graphs with average degree $\varepsilon n$, where $n$ denotes the number of vertices. Other ratios were previously studied. In a companion paper to the present paper, Camby 
and Schaudt [2] consider the Price of Connectivity for dominating set. Recently, Schaudt [12] studied the ratio between the connected domination number and the total domination number. Fulman [7] and Zverovich [15] investigated the ratio between the independence number and the upper domination number.

\section{Our results}

All the proofs can be found in the next section.

\subsection{Computational Complexity}

The class $\Theta_{2}^{P}=P^{\mathrm{NP}[\log ]}$ is defined as the class of decision problems solvable in polynomial time by a deterministic Turing machine that is allowed to use $\mathcal{O}(\log n)$ many queries to an NP-oracle, where $n$ is the size of the input.

Theorem 1 Let $1<r<2$ be a fixed rational number. Given a connected graph $G$, the problem of deciding whether $\tau_{c}(G) / \tau(G) \leq r$ is $\Theta_{2}^{p}$-complete.

It is easy to see that the above decision problem belongs to $\Theta_{2}^{p}$, since both $\tau$ and $\tau_{c}$ can be computed using logarithmically many queries to an NP-oracle by binary search. Thus, Theorem 1 is a negative result: loosely speaking, it tells us that deciding whether the PoC is bounded by some constant is as hard as computing both $\tau$ and $\tau_{c}$ explicitely. And this remains true even if the constant is not part of the input.

Our reduction is from the decision problem whether for two given graphs $G$ and $H$ it holds that $\tau(G) \geq$ $\tau(H)$, which is known to be $\Theta_{2}^{p}$-complete due to Spakowski and Vogel [13]. It uses a gadgetry that allows us to compare $\tau$ and $\tau_{c}$ on a single graph.

However, the problem of deciding of the $P o C$ is solved in polynomial time for certain class of graphs, for instance in the class of chordal graphs. Indeed, we knew that Escoffier, Gourvès and Monnot [4] proved that the problem of deciding about the connected vertex cover is polynomial in this class of graphs. Moreover, Rose, Lueker and Tarjan [11] show that an algorithm known as lexicographic breadth-first search solves the problem of deciding about the vertex cover in linear time by finding a perfect elimination ordering. Furthermore, they found an efficient recognition of chordal graph using this way.

Unfortunately, it seems that our machinery does not apply to the case of $r=1$. We leave it as an open problem to determine the computational complexity of the decision whether $\tau_{c}(G) / \tau(G)=1$ for a given graph $G$.

\subsection{PoC-Perfect Graphs}

As Theorem 1 shows, the class of graphs where $\tau_{c}(G) / \tau(G) \leq r$ holds (for any fixed rational $r \in(1,2)$ ) is $\Theta_{2}^{p}$-complete to recognize. However, if we restrict our attention to hereditary graph classes, we are able to derive the following results. Note that our characterizations yield polynomial time recognition algorithms, since the list of forbidden induced subgraphs is finite in each case.

We first consider the hereditary class of graphs $G$ for which $\tau_{c}(G)=\tau(G)$, referred to as PoC-Perfect graphs. A similar result had been found by Zverovich [14] for dominating set. There, the corresponding class is that of $\left(P_{5}, C_{5}\right)$-free graphs.

Theorem 2 The following assertions are equivalent for every graph $G$ :

(i) For every induced subgraph $H$ of $G$ it holds that $\tau_{c}(H)=\tau(H)$. 
(ii) $G$ is $\left(P_{5}, C_{5}, C_{4}\right)$-free.

(iii) $G$ is chordal and $P_{5}$-free.

By this characterization, the problem of deciding whether $\tau_{c}(H) / \tau(H)=1$ holds for every connected induced subgraph $H$ of a given graph is solved in polynomial time. In fact, this problem is equivalent to recognize a $P_{5}$-free and chordal graph. This problem is solvable in polynomial time clearly.

The above characterization tells us that the class of PoC-Perfect graphs properly contains two wellknown classes of graphs: split graphs and trivially perfect graphs (see [1] for further reference on these classes). Moreover, it gives rise to the following definition.

\subsection{PoC-Near-Perfect Graphs}

Let $t \in[1,2)$. A graph $G$ is said to be PoC-Near-Perfect with threshold $t$ if every induced subgraph $H$ of $G$ satisfies $\tau_{c}(H) \leqslant t \cdot \tau(H)$. This defines a hereditary class of graphs for every choice of $t$. Theorem 2 gives a forbidden induced subgraphs characterization of this class for $t=1$. Our second result gives such a characterization for $t=4 / 3$.

Note that $\tau_{c}\left(C_{5}\right) / \tau\left(C_{5}\right)=4 / 3$ and $\tau_{c}\left(P_{5}\right) / \tau\left(P_{5}\right)=\tau_{c}\left(C_{4}\right) / \tau\left(C_{4}\right)=3 / 2$. Hence any graph class that does not forbid either $C_{5}$ or $P_{5}$ contains a graph $G$ such that $\tau_{c}(G) / \tau(G)=4 / 3$. Therefore, the characterization of Theorem 2 also holds for the class of graphs $G$ such that every induced subgraph $H$ satisfies $\tau_{c}(H) \leqslant t \cdot \tau(H)$, for any $t \in[1,4 / 3)$. We now turn our attention to $t=4 / 3$, which is the next interesting threshold after $t=1$.

Theorem 3 The following assertions are equivalent for every graph $G$ :

(i) For every induced subgraph $H$ of $G$ it holds that $\tau_{c}(H) \leqslant \frac{4}{3} \cdot \tau(H)$.

(ii) $G$ is $\left(P_{5}, C_{4}\right)$-free.

By Theorem $3, t=3 / 2$ is the next interesting threshold after $t=4 / 3$. Our third results states that the list of forbidden induced subgraphs for threshold $t=3 / 2$ is $\left(C_{6}, P_{7}, \Delta_{1}, \Delta_{2}\right)$, where $\Delta_{1}$ is the 1-join of two $C_{4}$ 's, and $\Delta_{2}$ is obtained from $\Delta_{1}$ by removing one edge incident to the vertex of degree 4 (see Fig. 11).

Theorem 4 The following assertions are equivalent for every graph $G$ :

(i) For every induced subgraph $H$ of $G$ it holds that $\tau_{c}(H) \leqslant \frac{3}{2} \cdot \tau(H)$.

(ii) $G$ is $\left(P_{7}, C_{6}, \Delta_{1}, \Delta_{2}\right)$-free.

Since a chordal and $P_{7}$-free graph is $\left(C_{6}, P_{7}, \Delta_{1}, \Delta_{2}\right)$-free, we deduce the following corollary from Theorem 4

Corollary 1 If $G$ is a chordal, $P_{7}$-free graph then for every induced subgraph $H$ of $G$, it holds that $\tau_{c}(H) \leqslant 3 / 2 \cdot \tau(H)$. 

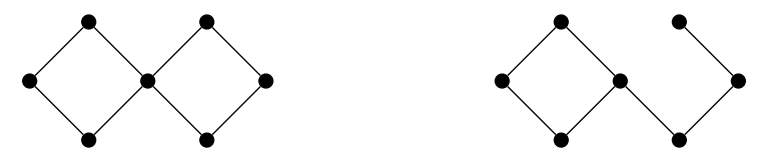

Fig. 1: An illustration of graphs $\Delta_{1}$ (on the left) and $\Delta_{2}$ (on the right).

\subsection{PoC-Critical Graphs}

We now turn our attention to critical graphs, that is, graphs $G$ for which the PoC of any proper induced subgraph $H$ of $G$ is strictly smaller than the PoC of $G$. These are exactly the graphs that can appear in a forbidden induced subgraphs characterization of the PoC-near-perfect graphs for some threshold $t \in[1,2)$. A perhaps more tractable class of graphs are the strongly critical graphs, defined as the graphs $G$ for which every proper (not necessarily induced) subgraph $H$ of $G$ has a PoC that is strictly smaller than the $\mathrm{PoC}$ of $G$. It is clear that every strongly critical graph is critical, but the converse is not true. For instance, $C_{5}$ is critical, but not strongly critical.

\subsubsection{PoC-Critical Chordal Graphs}

Let $T$ be a tree. We call $T$ special if it is obtained from another tree by subdividing each edge exactly once and then attaching a pendant vertex to every leaf of the resulting graph (see Fig. 2f for an example).

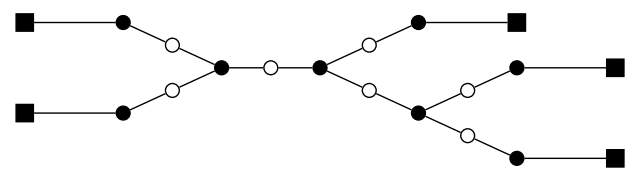

Fig. 2: A special tree constructed from another tree (vertices indicated by filled circles) by sudividing each edge exactly once (subdivision vertices are indicated by hollow circles) and by attaching a pendant vertex (indicated by squares) to every leaf of the resulting graph.

Our next result characterizes the class of (strongly) critical chordal graphs.

Theorem 5 For a chordal graph $G$, the following assertions are equivalent :

(i) $G$ is a special tree.

(ii) $G$ is strongly critical.

(iii) $G$ is critical.

\subsubsection{PoC-Strongly-Critical Graphs}

Our final result yields structural constraints on the class of strongly critical graphs.

Theorem 6 Let $G$ be a strongly critical graph.

(i) Every minimum vertex cover of $G$ is independent. In particular, $G$ is bipartite.

(ii) If $G$ has a cutvertex, then $G$ is a special tree. 


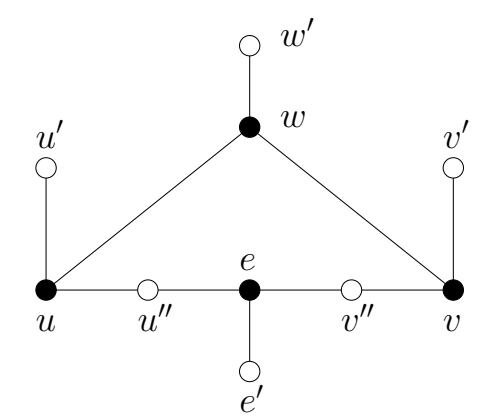

Fig. 3: Representation of an edge $e=u v$ in the construction of $G^{\prime}$ in Lemma 2 .

\section{Proofs}

\subsection{Complexity result}

We now proceed to prove Theorem 1

Lemma 1 Given a connected graph $G$ with $n$ vertices, one can construct in linear time a graph $G^{\prime}$ such that $\tau\left(G^{\prime}\right)=n+\tau(G)$ and $\tau_{c}\left(G^{\prime}\right)=2 n$.

Proof: With each vertex $v \in V(G)$, associate three vertices $v, v^{\prime}, v^{\prime \prime}$ in $V\left(G^{\prime}\right)$, and let $E\left(G^{\prime}\right):=E(G) \cup$ $\bigcup_{v \in V(G)}\left\{v v^{\prime}, v^{\prime} v^{\prime \prime}\right\}$. A minimum vertex cover of $G^{\prime}$ is the union of a minimum vertex cover of $G$ with all vertices of the form $v^{\prime}$. On the other hand, a minimum connected vertex cover of $G^{\prime}$ contains all vertices $v, v^{\prime}$.

Lemma 2 Given a graph $G$ with $n$ vertices and $m$ edges, one can construct in linear time a graph $G^{\prime}$ such that $\tau\left(G^{\prime}\right)=n+m+1$ and $\tau_{c}\left(G^{\prime}\right)=n+m+1+\tau(G)$.

Proof: For each edge $e=u v \in E(G)$, define two vertices $e, e^{\prime}$ of $V\left(G^{\prime}\right)$. For each vertex $v \in V(G)$, define three vertices $v, v^{\prime}, v^{\prime \prime}$ of $V\left(G^{\prime}\right)$. Finally, add two vertices $w, w^{\prime}$ to $V\left(G^{\prime}\right)$. The set of edges $E\left(G^{\prime}\right)$ is defined as follows. For each edge $e=u v \in E(G)$, the vertices $e$ and $e^{\prime}$ of $V\left(G^{\prime}\right)$ are adjacent, and vertex $e$ is adjacent to vertices $u^{\prime \prime}$ and $v^{\prime \prime}$. Similarly, for each vertex $v \in V(G)$, vertices $v$ and $v^{\prime}$ of $V\left(G^{\prime}\right)$ are adjacent, and $v$ is adjacent to both $v^{\prime \prime}$ and $w$. Finally, $w w^{\prime} \in E\left(G^{\prime}\right)$. The construction is illustrated in Figure 3 .

Since for each edge $e \in E(G)$, the corresponding vertex $e \in V\left(G^{\prime}\right)$ is adjacent to the degree-one vertex $e^{\prime}$, it can be considered, without loss of generality, to be part of any minimum vertex cover of $G^{\prime}$. The same remark holds for vertices $v \in V(G)$, and for the unique vertex $w$. Now the union $C \subset V\left(G^{\prime}\right)$ of those vertices is a vertex cover of $G^{\prime}$, hence we have $\tau\left(G^{\prime}\right)=n+m+1$.

We now have to compute $\tau_{c}\left(G^{\prime}\right)$. The previous vertex cover $C$ is not connected, as $G^{\prime}[C]$ has exactly $m+1$ connected components: one for each edge of $G$, and one induced by $w$ and the vertices $v \in V(G)$. To make it connected, we need to augment $C$ with the fewest possible additional vertices of the form $v^{\prime \prime}$ for $v \in V(G)$. Every such vertex $v^{\prime \prime}$ will link the component containing $v$ to every vertex $e \in E(G)$ of $G^{\prime}$ such that $v \in e$. Hence the minimum number of additional vertices to add to $C$ is exactly the size $\tau(G)$ of a minimum vertex cover of $G$. Thus $\tau_{c}\left(G^{\prime}\right)=n+m+1+\tau(G)$, as claimed. 
Proof of Theorem 1: Let $r=r_{1} / r_{2}$ be a fixed rational number with $1<r<2$. It is clear that the problem is in $\Theta_{2}^{p}$, so we proceed to the $\Theta_{2}^{p}$-hardness. Let $G$ and $H$ be two graphs. We reduce from the $\Theta_{2}^{p}$-complete decision problem, whether $\tau(G) \geq \tau(H)$ (see Spakowski and Vogel [13]).

We can assume that $G$ and $H$ are both connected. Otherwise, we choose a vertex from each connected component of $G$ (resp. $H$ ), add two new vertices $w$ and $w^{\prime}$, and put an edge from $w$ to all chosen vertices and to $w^{\prime}$. Let $G^{\prime}$ (resp. $H^{\prime}$ ) be the graph obtained from $G$ (resp. $H$ ) by this procedure. It is clear that $\tau\left(G^{\prime}\right)=\tau(G)+1$ and $\tau\left(H^{\prime}\right)=\tau(H)+1$. Hence, $\tau(G) \geq \tau(H)$ if and only if $\tau\left(G^{\prime}\right) \geq \tau\left(H^{\prime}\right)$. So we may assume that both $G$ and $H$ are connected.

The reduction consists of the following five steps.

Step 1. Let $v$ be any vertex of $G$. Starting with $r_{2}$ disjoint copies of $G$, we connect all $r_{2}$ copies of $v$ to a new vertex $w$. We then attach a pendant vertex $w^{\prime}$ to $w$. The graph obtained we denote by $G_{r_{2}}$. Let $n_{G}=|V(G)|$. Clearly, $\tau\left(G_{r_{2}}\right)=r_{2} \tau(G)+1$ and $\left|V\left(G_{r_{2}}\right)\right|=r_{2} n_{G}+2$.

Similarly we construct $H_{r_{1}}$ from $H$. Let $n_{H}=|V(H)|$ and $m_{H}=|E(H)|$. Clearly, $\tau\left(H_{r_{1}}\right)=$ $r_{1} \tau(H)+1,\left|V\left(H_{r_{1}}\right)\right|=r_{1} n_{H}+2$, and $\left|E\left(H_{r_{1}}\right)\right|=r_{1} m_{H}+r_{1}+1$.

Step 2. We apply Lemma 1 to $G_{r_{2}}$ to get $G_{r_{2}}^{\prime}$. We obtain

$$
\begin{aligned}
\tau\left(G_{r_{2}}^{\prime}\right) & =\left|V\left(G_{r_{2}}\right)\right|+\tau\left(G_{r_{2}}\right) \\
& =r_{2} \tau(G)+r_{2} n_{G}+3, \\
\tau_{c}\left(G_{r_{2}}^{\prime}\right) & =2\left|V\left(G_{r_{2}}\right)\right| \\
& =2 r_{2} n_{G}+4 .
\end{aligned}
$$

We apply Lemma 2 to $H_{r_{1}}$ to get $H_{r_{1}}^{\prime}$, and obtain

$$
\begin{aligned}
\tau\left(H_{r_{1}}^{\prime}\right) & =\left|V\left(H_{r_{1}}\right)\right|+\left|E\left(H_{r_{1}}\right)\right|+1 \\
& =r_{1}\left(n_{H}+m_{H}+1\right)+4 \\
\tau_{c}\left(H_{r_{1}}^{\prime}\right) & =\tau\left(H_{r_{1}}\right)+\left|V\left(H_{r_{1}}\right)\right|+\left|E\left(H_{r_{1}}\right)\right|+1 \\
& =r_{1} \tau(H)+r_{1}\left(n_{H}+m_{H}+1\right)+5 .
\end{aligned}
$$

Step 3. We construct a new graph $U$ by taking the disjoint union of $G_{r_{2}}^{\prime}$ and $H_{r_{1}}^{\prime}$, and adding an edge $u v$ such that $u \in V\left(G_{r_{2}}^{\prime}\right), v \in V\left(H_{r_{1}}^{\prime}\right)$, and both $u$ and $v$ are adjacent to a degree-one vertex in $G_{r_{2}}^{\prime}$ and $H_{r_{1}}^{\prime}$, respectively (such an edge always exists).

By construction of $U$,

$$
\begin{aligned}
\tau_{c}(U) & =\tau_{c}\left(G_{r_{2}}^{\prime}\right)+\tau_{c}\left(H_{r_{1}}^{\prime}\right) \\
& =r_{1} \tau(H)+r_{1}\left(n_{H}+m_{H}+1\right)+2 r_{2} n_{G}+9 \\
\tau(U) & =\tau\left(G_{r_{2}}^{\prime}\right)+\tau\left(H_{r_{1}}^{\prime}\right) \\
& =r_{2} \tau(G)+r_{1}\left(n_{H}+m_{H}+1\right)+r_{2} n_{G}+7
\end{aligned}
$$

Step 4. Let $\varphi_{1}=2 r_{2} n_{G}+r_{1}\left(n_{H}+m_{H}+1\right)+9$ and $\varphi_{2}=r_{2} n_{G}+r_{1}\left(n_{H}+m_{H}+1\right)+7$. In this step, we determine two non-negative integers $a$ and $b$ such that

$$
\frac{a+2 b+\varphi_{1}}{a+b+\varphi_{2}}=r .
$$


Let $p=\max \left\{\left|\varphi_{2}-\varphi_{1}\right|,\left|\varphi_{1}-2 \varphi_{2}\right|\right\}>0$. Let $a=p\left(2 r_{2}-r_{1}\right)+\varphi_{1}-2 \varphi_{2}$ and $b=p\left(r_{1}-r_{2}\right)+\varphi_{2}-\varphi_{1}$. Since $1<r<2$, note that $2 r_{2}-r_{1} \geqslant 1$ and $r_{1}-r_{2} \geqslant 1$. Therefore, $a$ and $b$ are non-negative integers. Now, we show that $a$ and $b$ satisfy (2). The numerator in (2) becomes $a+2 b+\varphi_{1}=p r_{1}$ whereas the denominator is $a+b+\varphi_{2}=p r_{2}$. Hence, the ratio equals to $r$. This proves our claim.

Step 5. We now construct a graph $U^{\prime}$ from $U$ as follows. Let $v$ be a vertex in $U$ of degree 1 (such a vertex is always present). Let $P^{1}$ be the graph obtained from the chordless path with vertex set $\left\{u_{1}, u_{2}, \ldots, u_{a}\right\}$ by attaching a pendant vertex to every member of $\left\{u_{1}, u_{2}, \ldots, u_{a}\right\}$. Let $P^{2}$ be the graph obtained from the chordless path with vertex set $\left\{v_{1}, v_{2}, \ldots, v_{2 b}\right\}$ by attaching a pendant vertex to every member of $\left\{v_{2}, v_{4}, \ldots, v_{2 b}\right\}$. Let $U^{\prime}$ be the graph obtained from the disjoint union of $U, P^{1}$, and $P^{2}$ by putting an edge from $v$ to $u_{1}$ and to $v_{1}$. Since $a, b \in \mathcal{O}\left(\varphi_{1}+\varphi_{2}\right)$, the above procedure can be done in linear time in the size of the graph $U$.

By the construction of $U^{\prime}$, we obtain

$$
\begin{aligned}
\tau_{c}\left(U^{\prime}\right) & =\tau_{c}(U)+a+2 b \\
& =r_{1} \tau(H)+a+2 b+\varphi_{1}, \\
\tau\left(U^{\prime}\right) & =\tau(U)+a+b \\
& =r_{2} \tau(G)+a+b+\varphi_{2} .
\end{aligned}
$$

Recall that $r=r_{1} / r_{2}$. By (2), there is some non-negative integer $c$ such that $a+2 b+\varphi_{1}=r_{1} c$ and $a+b+\varphi_{2}=r_{2} c$. Hence,

$$
\frac{\tau_{c}\left(U^{\prime}\right)}{\tau\left(U^{\prime}\right)}=\frac{r_{1} \tau(H)+a+2 b+\varphi_{1}}{r_{2} \tau(G)+a+b+\varphi_{2}}=\frac{r_{1} \tau(H)+r_{1} c}{r_{2} \tau(G)+r_{2} c}=r \frac{\tau(H)+c}{\tau(G)+c} .
$$

Thus, $\tau_{c}\left(U^{\prime}\right) / \tau\left(U^{\prime}\right) \leq r$ if and only if $\tau(H) \leq \tau(G)$. This completes the proof.

\subsection{Structural results}

Lemma 3 Let $G$ be a connected graph and let $C$ be a vertex cover of $G$. If $(\mathcal{A}, \mathcal{B})$ is a bipartition of the connected components of $C$ with $\mathcal{A}, \mathcal{B} \neq \emptyset$, there exists $A \in \mathcal{A}$ and $B \in \mathcal{B}$ such that the distance between $A$ and $B$ is exactly 2.

Proof: Let $(\mathcal{A}, \mathcal{B})$ be a bipartition of the connected components of $C$. Since $C$ has a finite number of connected components, there exist $A \in \mathcal{A}$ and $B \in \mathcal{B}$ such that the distance between them is minimum. Now we show that this distance is 2 . Otherwise, let $x_{1} x_{2} \ldots x_{n}$ be a shortest path between $A$ and $B$ with $x_{1} \in A$ and $x_{n} \in B$, where $n \geqslant 4$. In this case, no $x_{i}, i=2, \ldots, n-1$, belongs to $C$. Otherwise, $B$ is not one nearest component of $\mathcal{B}$ from $A$ or $A$ is not one nearest component of $\mathcal{A}$ from $B$. Thus, the edge $x_{2} x_{3}$ is not covered by $C$, in contradiction with the definition of vertex cover.

\subsection{PoC-Perfect graphs}

Proof Theorem 2: The class of graphs that are chordal and do not contain an induced $P_{5}$ is exactly the class of $\left(C_{4}, C_{5}, P_{5}\right)$-free graphs. Since $\tau_{c}\left(C_{4}\right) / \tau\left(C_{4}\right)=\tau_{c}\left(P_{5}\right) / \tau\left(P_{5}\right)=3 / 2$, and $\tau_{c}\left(C_{5}\right) / \tau\left(C_{5}\right)=$ $4 / 3$, any graph that contains $C_{4}, C_{5}$, or $P_{5}$ as an induced subgraph does not satisfy the first property. 
Hence it remains to show that every graph that does not satisfy the first property contains either a $C_{4}$, a $C_{5}$, or a $P_{5}$ as induced subgraph.

Consider a connected graph $G=(V, E)$, every minimum vertex cover of which induces at least two connected components. Pick such a minimum vertex cover $C \subset V$ that induces the smallest number of connected components. There must exist two subsets $A, B \subseteq C$ inducing two disjoint connected components, and a vertex $v$, such that $G[A \cup B \cup\{v\}]$ is connected, by Lemma 3 .

Consider the breadth-first search (BFS) trees $T_{A} \subseteq E$ in $G[\{v\} \cup A]$, and $T_{B} \subseteq E$ in $G[\{v\} \cup B]$, both rooted at $v$. If both trees have height at least two, then there is an induced $P_{5}$. Hence at least one of the trees, say $T_{B}$, has height one, that is, $N(v) \cap B=B$. Now we consider the set $C^{\prime}:=(C \backslash\{w\}) \cup\{v\}$, where $w$ is an arbitrary vertex of $B$. Since the number of connected components in $G\left[C^{\prime}\right]$ is strictly less than the number of connected components in $G[C]$, and $C^{\prime}$ is not bigger than $C$, the new set $C^{\prime}$ cannot be a vertex cover. Therefore, there must exist a vertex $x \notin C$, such that $w x \in E$ is not covered by $C^{\prime}$. Note that $x v \notin E$ (otherwise it would not be covered by $C$ ). If $x$ is adjacent to a vertex $t \in A$ that is itself adjacent to $v$, then we have found a $C_{4}$. If $x$ is adjacent to a vertex $t \in A$ that is not adjacent to $v$, then, using the shortest path from $v$ to $t$ in $T_{A}$, we find a cycle of length at least 5 .

Hence there remains the case where $x$ is not adjacent to any vertex in $A$. In that case, provided the height of $T_{A}$ is at least two, we can find a $P_{5}$. If the height of $T_{A}$ is exactly one, then $N(v) \cap A=A$, and we can do the same reasoning as above, and show there is a vertex $y \notin C$ adjacent to a vertex $z \in A$. Similarly, we can assume that $y$ is not adjacent to any vertex in $B$. Note that $x \neq y$ because of nonadjacency between $x$ and $A$ and $y z \in E$ with $z \in A$. Hence, the path going from $x$ to $y$ through $A, v$, and $B$ is an induced $P_{5}$.

\subsection{PoC-Near-Perfect graphs}

If $G$ is not connected, there always exists a connected component of $G$ such that the price of connectivity of $G$ is smaller than the $P o C$ of this connected component because $\frac{a+b}{c+d} \in\left[\frac{a}{c}, \frac{b}{d}\right]$, for any $a, b, c, d \in \mathbb{N}_{0}$. Therefore, we consider that $G$ is connected. Let $C$ be a vertex cover of a graph $G$. Let $C^{\prime}$ be the vertex set of a connected component of $G[C]$. We define $P_{C}\left(C^{\prime}\right)$ to be the set of vertices $v \in V(G)$ such that $N(v) \cap C \subseteq C^{\prime}$. It is clear that $C^{\prime} \subseteq P_{C}\left(C^{\prime}\right)$.

To prove Theorem 3 , we need to use the following lemma.

Lemma 4 Let $S_{1}, S_{2}, \ldots, S_{k}$ be the vertex sets of connected components of a vertex cover $C$. There exists at least one $P_{C}\left(S_{i}\right)$ which is not a cutset of $G$, i.e. $G\left[V(G) \backslash P_{C}\left(S_{i}\right)\right]$ is always connected.

Proof: We consider the new following graph $H$ defined by

$$
V(H)=\left\{P_{C}\left(S_{i}\right) \mid i=1, \ldots, k\right\}
$$

and

$$
E(H)=\left\{P_{C}\left(S_{i}\right) P_{C}\left(S_{j}\right) \mid N\left(P_{C}\left(S_{i}\right)\right) \cap N\left(P_{C}\left(S_{j}\right)\right) \neq \emptyset\right\} .
$$

Note that the sets $P_{C}\left(S_{i}\right), 1 \leqslant i \leqslant k$, are disjoint and induce a connected subgraph of $G$ each. Because $C$ is a vertex cover, $H$ is connected. Because every connected graph contains a no cutvertex, there exists at least one $P_{C}\left(S_{i}\right)$ which is not a cutvertex of $H$. Therefore, $P_{C}\left(S_{i}\right)$ is not a cutset of $G$. 
Proof Theorem 3: Since the PoC of $P_{5}$ and $C_{4}$ equals 3/2, any graph that contains $C_{4}$ or $P_{5}$ as an induced subgraph does not satisfy the first property. Hence, it remains to show that every graph that does not satisfy the first property contains either a $C_{4}$ or a $P_{5}$ as induced subgraph.

Let $G$ be a $\left(P_{5}, C_{4}\right)$-free graph. The proof is by induction on the number of components of a minimum vertex cover, say $k$. Let $C$ be such a vertex cover of $G$. Let $S_{1}, S_{2}, \ldots, S_{k}$ be the vertex set of the connected components of $G[C]$.

If $C$ is connected $(k=1)$, then $\tau_{c} / \tau=1$.

If $k=2$, i.e. $S_{1}$ and $S_{2}$ are connected components of $G[C]$, we have a vertex $x$ adjacent to $S_{1}$ and $S_{2}$, by Lemma 3 . Hence, $\tau_{c}(G) \leqslant \tau(G)+1$. Because $G$ does not satisfy the first property of the theorem, $\tau(G)=2$, i.e. $S_{1}=\left\{s_{1}\right\}$ and $S_{2}=\left\{s_{2}\right\}$. If $s_{1}$ and $s_{2}$ have at least two common neighbors, there is an induced $C_{4}$. Otherwise $x$ is the unique common neighbor of $s_{1}$ and $s_{2}$. Because $\tau_{c}(G) \neq 2=\tau(G)$, each connected component has at least one private neighbour, i.e. there exist $x_{1}, x_{2} \notin C \cup\{x\}$ with $x_{1} s_{1}, x_{2} s_{2} \in E$. Note that $x_{1} x_{2} \notin E$ because $C$ is a vertex cover and $x_{1}, x_{2} \notin C$. Therefore $G\left[\left\{x_{1}, s_{1}, x, s_{2}, x_{2}\right\}\right]$ is an induced $P_{5}$.

If $S_{1}, S_{2}$ and $S_{3}$ are connected components of $G[C]$, we can suppose, without loss of generality, that there exist $x_{1} \in N\left(S_{1}\right) \cap N\left(S_{2}\right)$ and $x_{2} \in N\left(S_{2}\right) \cap N\left(S_{3}\right)$, by Lemma 3 . The vertex $x_{2}$ is adjacent to $S_{1}$ (or $x_{1}$ is adjacent to $S_{3}$ ), otherwise $G\left[C \cup\left\{x_{1}, x_{2}\right\}\right]$ contains a $P_{5}$. Thus, $C \cup\left\{x_{2}\right\}$, resp. $C \cup\left\{x_{1}\right\}$, is a connected vertex cover. Hence,

$$
\frac{\tau_{c}(G)}{\tau(G)} \leqslant \frac{|C|+1}{|C|} \leqslant \frac{4}{3} .
$$

Now $k \geqslant 4$ and we assume that $\tau_{c} \leqslant 4 / 3 \tau$ holds for every connected $\left(P_{5}, C_{4}\right)$-free graph with a minimum vertex cover of at most $k-3$ connected components. Let $S_{1}, S_{2}, S_{3}, \ldots S_{k}$ be the vertex sets of the connected components of $G[C]$. By Lemma 4 at least one of these sets, say $P_{C}\left(S_{3}\right)$, is not a cutset of $G$. By applying twice the Lemma 4 , two more of these sets, say $P_{C}\left(S_{2}\right)$, resp. $P_{C}\left(S_{1}\right)$, are not a cutset of $G\left[V \backslash P_{C}\left(S_{3}\right)\right]$, resp. $G\left[V \backslash\left(P_{C}\left(S_{2}\right) \cup P_{C}\left(S_{3}\right)\right)\right]$. Let $C^{\prime}=C \backslash\left(S_{1} \cup S_{2} \cup S_{3}\right)$ and note that $C^{\prime}$ is a minimum vertex cover of $G^{\prime}=G\left[V \backslash\left(P_{C}\left(S_{1}\right) \cup P_{C}\left(S_{2}\right) \cup P_{C}\left(S_{3}\right)\right)\right]$. By the induction hypothesis, there is a minimum connected vertex cover of $G^{\prime}$, say $C_{c}^{\prime}$, with $\left|C_{c}^{\prime}\right| \leqslant 4 / 3\left|C^{\prime}\right|$.

We show that there exists a connected vertex cover $C_{c}$ of $G$ with $\left|C_{c}\right| \leqslant\left|S_{1}\right|+\left|S_{2}\right|+\left|S_{3}\right|+\left|C_{c}^{\prime}\right|+1$, built from $S_{1}, S_{2}, S_{3}$ and $C_{c}^{\prime}$. Indeed, we have

$$
\frac{\tau_{c}(G)}{\tau(G)} \leqslant \frac{\left|S_{1}\right|+\left|S_{2}\right|+\left|S_{3}\right|+1+\left|C_{c}^{\prime}\right|}{\left|S_{1}\right|+\left|S_{2}\right|+\left|S_{3}\right|+\left|C^{\prime}\right|} \leqslant \max \left(\frac{\left|S_{1}\right|+\left|S_{2}\right|+\left|S_{3}\right|+1}{\left|S_{1}\right|+\left|S_{2}\right|+\left|S_{3}\right|}, \frac{\left|C_{c}^{\prime}\right|}{\left|C^{\prime}\right|}\right) \leqslant \frac{4}{3} .
$$

We refer to $C_{c}^{\prime}$ as $S_{4}$ for ease of writing. We observe that the set $V(G) \backslash\left(S_{1} \cup S_{2} \cup S_{3} \cup S_{4}\right)$ is an independent set because its complement is a vertex cover of $G$. We complete the proof with the following case distinction.

Case 1. There exists one component, say $S_{1}$, such that the other connected components are a distance 2 from $S_{1}$. Let $x_{i}$ be a vertex adjacent to $S_{1}$ and $S_{i}$, for $i=2,3,4$.

Case 1.1. It holds that $x_{2}=x_{3}=x_{4}$. We have immediately one vertex to connect $S_{1}, S_{2}, S_{3}$ and $S_{4}$.

Case 1.2. Two of the $x_{i}$ are equal, and the third one is distinct from them. We can suppose without loss of generality that $x_{3}=x_{4}$. We suppose by the previous case that $x_{2}$ is not adjacent to $S_{3} \cup S_{4}$ and $x_{3}$ is not adjacent to $S_{2}$. The path $S_{2} x_{2} S_{1} x_{3} S_{3}$ forms again a $P_{5}$. If there is an edge between $x_{3}$ and $S_{2}$, we take $x_{3}$ to connect $S_{1}, S_{2}, S_{3}$ and $S_{4}$. Otherwise, there must be an edge between $x_{2}$ and $S_{3}$. But then, we have an induced $P_{5}$ in $G\left[S_{2} \cup S_{3} \cup S_{4} \cup\left\{x_{2}, x_{3}\right\}\right]$, a contradiction. 
Case 1.3. The $x_{i}$ are mutually distinct and $x_{i}$ is not adjacent to $S_{j}$ for $i \neq j$ (otherwise we are in the previous cases). Since $G\left[S_{1} \cup S_{3} \cup S_{4} \cup\left\{x_{3}, x_{4}\right\}\right]$ contains an induced $P_{5}$, we are in the next case.

Case 2. Up to a renaming of the $S_{i}$, the distance between $S_{i}$ and $S_{i+1}$ is $2, i=1,2,3$. Let $x_{i}$ be a vertex adjacent to $S_{i}$ and $S_{i+1}, i=1,2,3$. Because $G$ is $P_{5}$-free, $S_{1}$ must be adjacent to $x_{2}$ or $x_{1}$ must be adjacent to $S_{3}$. Hence, we are in Case 1 .

To prove Theorem 4 , we need to use the following lemma.

Lemma 5 Let $G$ be a $\left(C_{6}, P_{7}, \Delta_{1}, \Delta_{2}\right)$-free graph, let $C$ be a vertex cover of $G$ such that $G[C]$ has exactly three connected components, and let $G$ contain an induced cycle of length 7 intersecting all connected components of $G[C]$. Then there exists a connected vertex cover $C_{c}$ such that

$$
\begin{array}{ll}
\left|C_{c}\right| \leqslant|C|+1 & \text { if }|C|>4, \\
\left|C_{c}\right|=6 & \text { if }|C|=4 .
\end{array}
$$

Proof: Let $x_{1} x_{2} x_{3} x_{4} x_{5} x_{6} x_{7}$ be an induced cycle intersecting the three connected components of $C$, say $S_{1}, S_{2}$ and $S_{3}$. Without loss of generality, we can suppose $x_{1} \in S_{1}, x_{3} \in S_{2}$ and $x_{5}, x_{6} \in S_{3}$ (see Fig 4 . We can assume that no vertex is adjacent to $S_{1}, S_{2}$ and $S_{3}$, otherwise the result is obviously true.

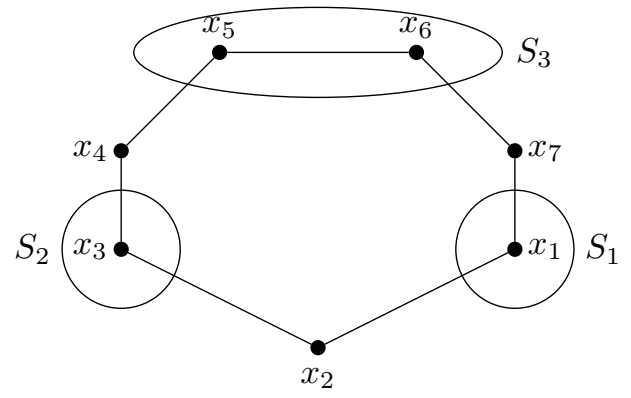

Fig. 4: $\mathrm{G}$ contains an induced $C_{7}$ intersecting all components of a vertex cover.

If $\left|N\left(x_{1}\right) \cap N\left(x_{3}\right)\right| \geqslant 2,\left|N\left(x_{1}\right) \cap N\left(x_{6}\right)\right| \geqslant 2$, or $\left|N\left(x_{5}\right) \cap N\left(x_{3}\right)\right| \geqslant 2$, then we have an induced subgraph $\Delta_{2}$.

Otherwise $N\left(x_{1}\right) \cap N\left(x_{3}\right)=\left\{x_{2}\right\}$ and $N\left(x_{1}\right) \cap N\left(x_{6}\right)=\left\{x_{7}\right\}$ and $N\left(x_{5}\right) \cap N\left(x_{3}\right)=\left\{x_{4}\right\}$. We distinguish several cases, depending on the cardinality of $S_{3}$.

The first case is $\left|S_{3}\right|=2$. If $\left|S_{1}\right|=\left|S_{2}\right|=1$, i.e., $|C|=4$, we have the connected vertex cover $S_{1} \cup S_{2} \cup S_{3} \cup\left\{x_{2}, x_{4}\right\}$ of six vertices.

We can suppose $\left|S_{2}\right|>1$. Then every vertex of $S_{2}$ is adjacent to both $x_{2}$ and $x_{4}$. Indeed, if an edge $y z$ of $S_{2}$ has one endvertex, say $y$, adjacent to both $x_{2}$ and $x_{4}$, then $z$ must be adjacent to both $x_{2}$ and $x_{4}$, otherwise $G$ contains a $P_{7}$. Let $x$ a vertex of $S_{2} \backslash\left\{x_{3}\right\}$ which is not a cutvertex of $G\left[S_{2}\right]$, i.e. $Y=S_{1} \cup S_{3} \cup\left(S_{2} \backslash\{x\}\right) \cup\left\{x_{2}, x_{4}\right\}$ induces a connected graph. If $Y$ is not a vertex cover, there exists a vertex $t \notin Y$ adjacent to $x$. Note that $t$ is distinct from $x_{7}$, because no vertex is adjacent to $S_{1}, S_{2}$ and $S_{3}$, and $t$ is not adjacent to $x_{1}$ or $x_{5}$, because $G$ is $\Delta_{2}$-free. Moreover $t$ is not adjacent to $x_{6}$ since $G$ is $C_{6}$-free. Therefore we have an induced $P_{7}$ subgraph (see Fig 5 ).

In the second case, $\left|S_{3}\right|>2$. If there exists a vertex $p_{1}$ in $S_{3} \backslash\left\{x_{5}, x_{6}\right\}$ which is adjacent to neither $x_{4}$ nor $x_{7}$, we claim that there is an induced $P_{7}$ subgraph. Indeed, the distance in $S_{3} \cup\left\{x_{4}, x_{7}\right\}$ between 


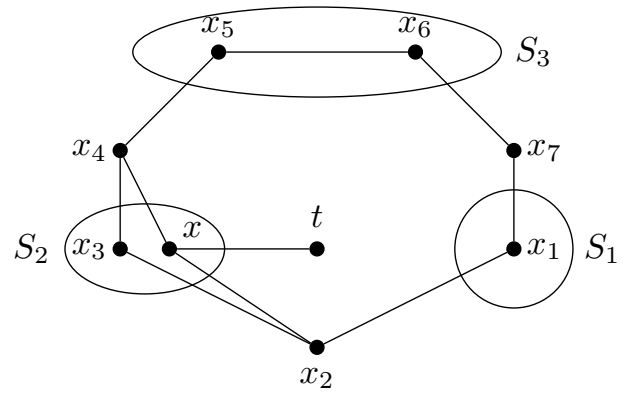

Fig. 5: G contains an induced $C_{7}$ and $P_{7}$ intersecting all components of a vertex cover.

this vertex $p_{1}$ and $x_{4}$, resp. $p_{1}$ and $x_{7}$, is at least 2 . Without lost of generality, we suppose that $p_{1}$ is closer to $x_{4}$ than $x_{7}$. Let $p_{1} p_{2} \ldots p_{s} x_{4}$ the shortest path between $p_{1}$ and $x_{4}$ through $S_{3}$. By definition, for $i=1, \ldots, s-1, p_{i}$ is adjacent neither to $x_{4}$ nor $x_{7}$. Note that $p_{s}$ is not adjacent to $x_{7}$ because $G$ is $C_{6}$-free. Hence, $G\left[\left\{x_{7}, x_{1}, x_{2}, x_{3}, x_{4}, p_{s} \ldots, p_{2}, p_{1}\right\}\right]$ contains an induced $P_{7}$. Let $y \in S_{3} \backslash\left\{x_{5}, x_{6}\right\}$ such that $y$ is not a cutvertex of $G\left[S_{3}\right]$. We can suppose that $y$ is adjacent to $x_{4}$. Because $G$ is $C_{6}$-free, $y$ is not adjacent to $x_{7}$. If $Y=S_{1} \cup S_{2} \cup\left(S_{3} \backslash\{y\}\right) \cup\left\{x_{7}, x_{4}\right\}$ is not a vertex cover, there exists a vertex $t \notin Y$ adjacent to $y$. Note that $t$ is distinct from $x_{2}$, because no vertex is adjacent to $S_{1}, S_{2}$ and $S_{3}$ (see Fig 6. If $t$ is adjacent to $x_{1}$ (resp. $x_{3}$ ), we have an induced $C_{6} \operatorname{subgraph}\left(\right.$ resp. $\Delta_{2}$ ).

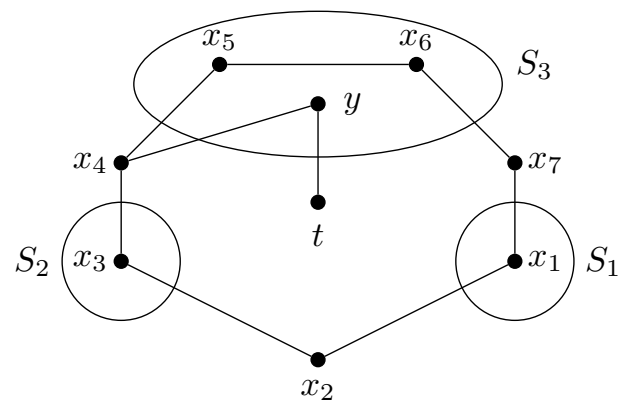

Fig. 6: $Y=S_{1} \cup S_{2} \cup\left(S_{3} \backslash\{y\}\right) \cup\left\{x_{4}, x_{7}\right\}$ is not a vertex cover of $G$.

Otherwise we have an induced $P_{7}$ subgraph.

Proof Theorem 4; If $G$ contains one of the four forbidden induced subgraphs, say $H$, then $\tau_{c}(H) / \tau(H)=$ $5 / 3$. It remains to prove that the Price of Connectivity of a $\left(P_{7}, C_{6}, \Delta_{1}, \Delta_{2}\right)$-free graph is bounded by $3 / 2$. So let $G$ be a $\left(P_{7}, C_{6}, \Delta_{1}, \Delta_{2}\right)$-free graph. The proof is by induction on the number of connected components of a minimum vertex cover. Let $C$ be a minimum vertex cover of $G$ with the minimum number of connected components, say $k$.

If $C$ is connected $(k=1)$, then $\tau_{c} / \tau=1$.

If $k=2$, by Lemma 3 .

$$
\tau_{c} / \tau \leqslant \frac{|C|+1}{|C|} \leqslant 1+\frac{1}{|C|} \leqslant 1+\frac{1}{2}=\frac{3}{2} .
$$




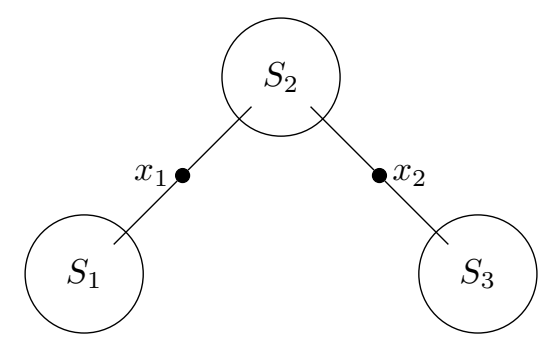

(a) Initial case

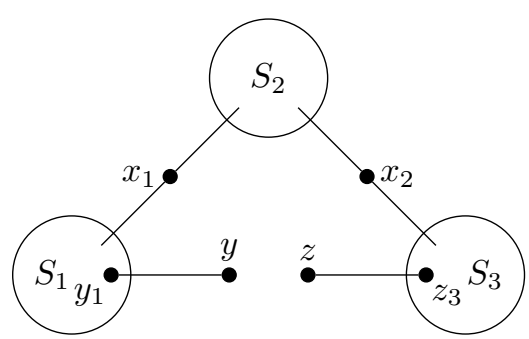

(b) Two private edges of a vertex cover

Fig. 7: Three components of a vertex cover to connect by adding only one vertex.

Now let $k \geqslant 3$. We may assume that $\tau_{c} \leqslant 3 / 2 \tau$ holds for every $\left(P_{7}, C_{6}, \Delta_{1}, \Delta_{2}\right)$-free graph with a minimum vertex cover of at most $k-2$ connected components. Let $S_{1}, S_{2}, S_{3}, \ldots, S_{k}$ be the vertex set of the connected components of $G[C]$. By Lemma 4, we may assume that the set $P_{C}\left(S_{2}\right)$, is not a cutset of $G$, and that the set $P_{C}\left(S_{1}\right)$ is not a cutset of $G^{\prime}=G\left[V \backslash P_{C}\left(S_{2}\right)\right]$. Note that the set $C^{\prime}=C \backslash\left(S_{1} \cup S_{2}\right)$ is a minimum vertex cover of the graph $G^{\prime \prime}=G\left[V \backslash\left(P_{C}\left(S_{1}\right) \cup P_{C}\left(S_{2}\right)\right)\right]$. By the induction hypothesis, there is a minimum connected vertex cover of $G^{\prime \prime}$, say $C_{c}^{\prime}$, with $\left|C_{c}^{\prime}\right| \leqslant 3 / 2\left|C^{\prime}\right|$.

We show that there exists a connected vertex cover $C_{c}$ of $G$ such that $\left|C_{c}\right| \leqslant\left|S_{1}\right|+\left|S_{2}\right|+\left|C_{c}^{\prime}\right|+1$, built from $S_{1}, S_{2}$ and $C_{c}^{\prime}$. Indeed, we have

$$
\frac{\tau_{c}(G)}{\tau(G)} \leqslant \frac{\left|S_{1}\right|+\left|S_{2}\right|+1+\left|C_{c}^{\prime}\right|}{\left|S_{1}\right|+\left|S_{2}\right|+\left|C^{\prime}\right|} \leqslant \max \left(\frac{\left|S_{1}\right|+\left|S_{2}\right|+1}{\left|S_{1}\right|+\left|S_{2}\right|}, \frac{\left|C_{c}^{\prime}\right|}{\left|C^{\prime}\right|}\right) \leqslant \frac{3}{2} .
$$

We refer to the set $C_{c}^{\prime}$ as $S_{3}$ for the ease of writing. We can suppose that there does not exist any single vertex to connect $S_{1}, S_{2}$, and $S_{3}$, otherwise the result is obviously true. Without loss of generality, there is a vertex $x_{i}$ adjacent only to $S_{i}$ and $S_{i+1}, i=1,2$, such that $x_{1} \neq x_{2}$ (see Fig.7a).

Note that $x_{1}$ and $x_{2}$ are not adjacent because $C$ is a vertex cover. Let $y_{1} \in S_{1}$ and $z_{3} \in S_{3}$ be two vertices such that $y_{1}$ and $z_{3}$ are not cutvertices in $G\left[S_{1} \cup S_{2} \cup S_{3} \cup\left\{x_{1}, x_{2}\right\}\right]$. If $S_{1} \cup S_{2} \cup\left(S_{3} \backslash\left\{z_{3}\right\}\right) \cup$ $\left\{x_{1}, x_{2}\right\}$ or $\left(S_{1} \backslash\left\{y_{1}\right\}\right) \cup S_{2} \cup S_{3} \cup\left\{x_{1}, x_{2}\right\}$ is a vertex cover, then $\tau_{c}(G) / \tau(G) \leqslant 3 / 2$. Thus, there exist two edges, say $y_{1} y$ and $z_{3} z$, with $y, z \notin S_{1} \cup S_{2} \cup S_{3} \cup\left\{x_{1}, x_{2}\right\}$ (see Fig. 7b). Note that $y$ can be equal to $z$.

Now, we discuss on the adjacency of $y$ with $S_{3}$ and $S_{2}$.

Case 1. The vertex $y$ is adjacent to $S_{3}$. Thus, $y$ is not adjacent to $S_{2}$. If the shortest induced cycle via $S_{1} \cup S_{2} \cup S_{3} \cup\left\{x_{1}, x_{2}, y\right\}$ is of length 6 or more than 8 , we have an induced $C_{6}$ or a $P_{7}$. Thus the shortest induced cycle via the three connected components has 7 vertices. By Lemma 5 , it is clear that $\tau_{c}(G) / \tau(G) \leqslant 3 / 2$ if $\left|S_{1}\right|+\left|S_{2}\right|+\left|S_{3}\right|>4$. Otherwise $S_{1}, S_{2}$ and $S_{3}$ are three connected components of the initial vertex cover $C$. Thus, by Lemma $5, \tau_{c}(G) / \tau(G) \leqslant 6 / 4=3 / 2$.

The cases that $z$ is adjacent to $S_{1}$ or $y=z$ are dealt with similarly.

Case 2. The vertex $y$ is adjacent to $S_{2}$ and $z$ is not adjacent to $S_{2}$. Since $G\left[S_{1} \cup S_{2} \cup S_{3} \cup\left\{x_{1}, x_{2}, y, z\right\}\right]$ does not contain $P_{7}$, there exists $t \in N\left(x_{1}\right) \cap N\left(x_{2}\right) \cap S_{2}$ and $t$ is adjacent to $y$. Hence, we have an induced $\Delta_{2}$.

Case 3. Both $y$ and $z$ are adjacent to $S_{2}$. Thus $y$ (resp. $z$ ) is not adjacent to $S_{3}$ (resp. $S_{1}$ ). Let $P$ be a shortest path from $z$ to $y$ that goes through $S_{3},\left\{x_{2}\right\}, S_{2},\left\{x_{1}\right\}$, and $S_{1}$. If $P$ has 7 vertices, then we have an induced $P_{7}, \Delta_{1}$ or $\Delta_{2}$ subgraph, depending on the adjacency of $y$ and $z$ with $S_{2}$. If $P$ contains at least 


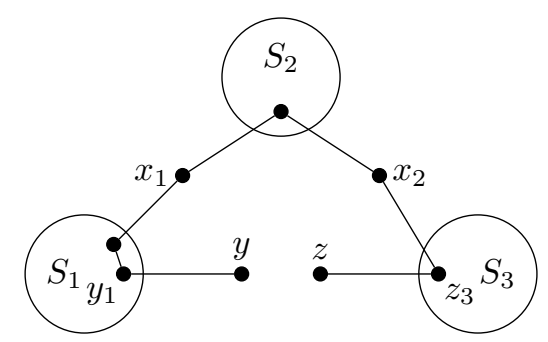

(a) Case 3.1.

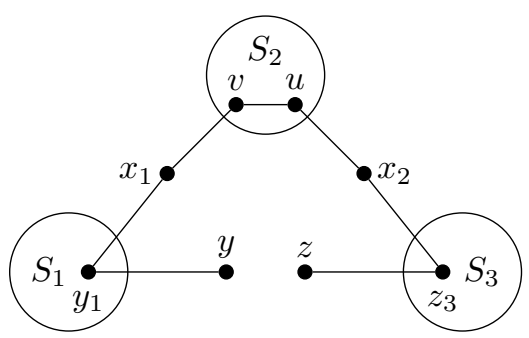

(b) Case 3.2.

Fig. 8: Three components of a vertex cover to connect by adding only one vertex.

nine vertices, we have an induced $P_{7}$ subgraph in $G\left[S_{1} \cup S_{2} \cup S_{3} \cup\left\{x_{1}, x_{2}\right\}\right]$. Otherwise $P$ has exactly 8 vertices. There are two possibilities.

Case 3.1. $S_{1}$ (or $S_{3}$ ) contains an edge of $P$ (see fig. 8a. Thus we have an induced $P_{7}$ or $\Delta_{2}$ in $\left.G\left[\left\{z, x_{1}, x_{2}\right\} \cup S_{1} \cup S_{2} \cup S_{3}\right\}\right]$, depending on the adjacency between $S_{2}$ and $z$.

Case 3.2. $S_{2}$ contains an edge of $P$ (see Fig. 8b, say $v u$. Then, if $z$ is not adjacent to $v, G$ contains a $P_{7}$ or a $\Delta_{2}$, depending on the adjacency between $z$ and $u$. Thus $z$ is adjacent to $v$. Hence, we have an induced $P_{7}$ or $\Delta_{2}$ subgraph in $G\left[S_{1} \cup\left(S_{2} \backslash\{u\}\right) \cup S_{3} \cup\left\{x_{1}, x_{2}, y, z\right\}\right]$, depending on the adjacency between $y$ and $v$.

Case 4. The vertex $y$ is adjacent to neither $S_{2}$ nor $S_{3}$. We can suppose that $z$ is adjacent to neither $S_{1}$ nor $S_{2}$ (thus $y \neq z$ ). Thus, $G$ contains a $P_{7}$.

\subsection{PoC-Critical Graphs}

\subsubsection{PoC-Critical Chordal Graphs}

Lemma 6 Let $G$ be a critical graph. For every minimum vertex cover $C$ of $G$, there does not exist a bridge of $G$ with endvertices in $C$.

Proof: Suppose there exists a bridge $x y$ with $x, y \in C$. The removal of the edge $x y$ results in two connected subgraphs of $G$, which we denote by $G_{1}$ resp. $G_{2}$. We can assume that $x \in V\left(G_{1}\right)$ and $y \in V\left(G_{2}\right)$. Let $G_{1}^{\prime}$ be the graph obtained from $G_{1}$ by attaching a pendant vertex to $x$. Analogously let $G_{2}^{\prime}$ be the graph obtained from $G_{2}$ by attaching a pendant vertex to $y$.

We observe that $C \cap V\left(G_{1}\right)$ is a vertex cover of $G_{1}^{\prime}$ and $C \cap V\left(G_{2}\right)$ is a vertex cover of $G_{2}^{\prime}$. Thus

$$
\tau(G) \geqslant \tau\left(G_{1}^{\prime}\right)+\tau\left(G_{2}^{\prime}\right) .
$$

On the other hand, let $C_{c, 1}$ be a connected vertex cover of $G_{1}^{\prime}$ and $C_{c, 2}$ be a connected vertex cover of $G_{2}^{\prime}$. We can assume that $C_{c, 1} \subseteq V\left(G_{1}\right)$ and $C_{c, 2} \subseteq V\left(G_{2}\right)$. It is clear that $x \in C_{c, 1}$ and $y \in C_{c, 2}$. Thus $C_{c, 1} \cup C_{c, 2}$ is a connected vertex cover of $G$. Since $C_{c, 1} \cap C_{c, 2}=\emptyset$,

$$
\tau_{c}(G) \leqslant \tau_{c}\left(G_{1}^{\prime}\right)+\tau_{c}\left(G_{2}^{\prime}\right) .
$$

But (3) and (4) say that

$$
\tau_{c}(G) / \tau(G) \leqslant \max \left\{\tau_{c}\left(G_{1}^{\prime}\right) / \tau\left(G_{1}^{\prime}\right), \tau_{c}\left(G_{2}^{\prime}\right) / \tau\left(G_{2}^{\prime}\right)\right\} .
$$


Since both $G_{1}^{\prime}$ and $G_{2}^{\prime}$ are isomorphic to induced subgraphs of $G, 5$ is a contradiction to the choice of $G$ to be critical.

Proof Theorem 5: It is obvious that (ii) implies (iii). First, we prove that (iii) implies (i), that is, every critical chordal graph is a special tree. For this, let $G$ be a critical chordal graph.

If the chordal graph $G$ is not a tree, then $G$ contains a triangle and every minimum vertex cover of $G$ contains at least two vertices of this triangle. Let $v$ be a vertex that is both in the triangle and in a minimum vertex cover. Then we have $\tau(G)=\tau(G-v)+1$ and also $\tau_{c}(G) \leqslant \tau_{c}(G-v)+1$, implying that $G$ is not critical. Therefore, $G$ is a tree.

Let $C$ be a minimum vertex cover of $G$.

First we show that $C$ is an independent set. Suppose there are $x, y \in C$ such that $x y \in E$. Since $G$ is a tree, $x y$ is a bridge, a contradiction with Lemma 6 .

Now we show that every member of $V \backslash C$ has degree at most two. For this, let $x \in V \backslash C$. Suppose that $|N(x)| \geqslant 3$. Let $X_{1}, X_{2}, \ldots, X_{k}$ be the vertex sets of the connected components of $G-x$. By assumption, $k \geqslant 3$. Let

$$
H_{1}=G-\bigcup_{i=3}^{k} X_{i}
$$

and

$$
H_{2}=G-\left(X_{1} \cup X_{2}\right)
$$

We observe that

$$
\tau(G) \geqslant \tau\left(H_{1}\right)+\tau\left(H_{2}\right)
$$

Since $x$ is a cutvertex of $H_{1}, x$ is contained in every connected vertex cover of $H_{1}$. Therefore

$$
\tau_{c}(G) \leqslant \tau_{c}\left(H_{1}\right)+\tau_{c}\left(H_{2}\right)
$$

By the same argumentation from Lemma 6, (6) and (7) yield a contradiction to the choice of $G$ to be critical. This proves that every vertex of $V \backslash C$ has at most two neighbors. By the discussion above, $C$ is an independent set and $G$ is a tree. Moreover, the degree of every vertex in $C$ is at least two. Otherwise let $v$ be a vertex of $C$ with degree 1 and let $u$ be the neighbor of $v$. Because $C$ is independent, $u \notin C$. Because $C$ is a vertex cover, every neighbor of $u$ is in $C$. Thus, $Y=(C \backslash\{v\}) \cup\{u\}$ is a minimum vertex cover but $Y$ is not independent, a contradiction. We prove that $G$ is a special tree. In fact, the initial tree $H$ is defined as following : $V(H)=C$ and $E(H)=\left\{u v \mid\right.$ there exists a path $P_{u v}$ in $(V(G) \backslash C) \cup\{v\}$ from $u$ to $v\}$. Because $C$ is a vertex cover of $G$, if $u v$ is an edge in $H$, then the length of the path $P_{u v}$ in $G$ is exactly 2 . Moreover, two 1 -degree vertices cannot have the same neighbor, because $G$ is critical. All in all, $G$ is a special tree.

Now, we show that (i) implies (ii), that is, every special tree is strongly critical. Let $G$ be a special tree. It is easy to see that $\tau_{c}(G) / \tau(G)=2-1 / \tau(G)$. If $G$ is not strongly critical, then there exists a proper subgraph $H$ of $G$ such that $\tau_{c}(H) / \tau(H) \geqslant \tau_{c}(G) / \tau(G)$. We can suppose that such an $H$ is minimal for inclusion. Thus $H$ is critical. By the previous argumentation, $H$ is a special tree. Therefore, $\tau_{c}(H) / \tau(H)=2-1 / \tau(H)$, but $2-1 / \tau(G)>2-1 / \tau\left(H^{\prime}\right)$ for every proper special subtree $H^{\prime}$ of $G$, a contradiction. This completes the proof. 


\subsubsection{PoC-Strongly-Critical Graphs}

Theorem 6 follows from Lemma 7 and Lemma 8 presented below.

Lemma 7 Let $G$ be a strongly critical graph. Then every minimum vertex cover of $G$ is an independent set. In particular, $G$ is bipartite.

Proof: Let $G$ be a strongly critical graph and let $C$ be a minimum vertex cover of $G$. Suppose that $C$ is not an independent set. Thus there are two adjacent vertices in $C$, say $x$ and $y$.

By Lemma 6, $x y$ cannot be a bridge of $G$. So $G-x y$ is connected. Let $C_{c}$ be a minimum connected vertex cover of $G-x y$. Suppose that $\{x, y\} \cap C_{c} \neq \emptyset$. Then $\tau_{c}(G-x y)=\tau_{c}(G)$, in contradiction to the choice of $G$ to be strongly critical. Thus $\{x, y\} \cap C_{c}=\emptyset$. Hence $C_{c} \cup\{x\}$ is a minimum connected vertex cover of $G$ and, moreover, $y \notin C_{c} \cup\{x\}$.

Let $A=N_{G}(y) \cap C$ and $B=N_{G}(y) \backslash C$. As $x \in A, A \neq \emptyset$. Since $C$ is a minimum vertex cover, $B \neq \emptyset$. Let $G^{\prime}$ be the graph obtained from $G$ by the removal of all edges joining $y$ to $B$. Since $C_{c} \cup\{x\}$ is a connected vertex cover of $G$ and $y \notin C_{c}$, then $A \cup B \subseteq C_{c} \cup\{x\}$ and $G^{\prime}$ is connected. As $C \backslash\{y\}$ is a vertex cover of $G^{\prime}, \tau\left(G^{\prime}\right)<\tau(G)$. Thus, by the choice of $G, \tau_{c}\left(G^{\prime}\right) \leqslant \tau_{c}(G)-2$. Let $C_{c}^{\prime}$ be a minimum vertex cover of $G^{\prime}$. Then $A \cap C_{c}^{\prime} \neq \emptyset$. Therefore $C_{c}^{\prime} \cup\{y\}$ is a connected vertex cover of $G$, in contradiction to the fact that $\left|C_{c}^{\prime} \cup\{y\}\right| \leqslant \tau_{c}\left(G^{\prime}\right)+1 \leqslant \tau_{c}(G)-1$. This completes the proof.

Lemma 8 Let $G$ be a strongly critical graph. If $G$ has a cutvertex, it is a special tree.

Proof: Let $G=(V, E)$ be a strongly critical graph with a cutvertex. Suppose that $G$ is not a tree. Thus $G$ has a non-trivial block. We can pick a cutvertex $x$ and an edge $e$ incident to $x$ in this block. The graph $G-e$ is connected, by the choice of $e$. Every connected vertex cover of $G-e$ contains $x$, as $x$ is a cutvertex of $G-e$. Hence, every connected vertex cover of $G-e$ covers $e$. Thus $\tau_{c}(G-e) \geqslant \tau_{c}(G)$, in contradiction to the choice of $G$ to be strongly critical. Hence, $G$ is a tree. In particular, $G$ is chordal.

The conclusion then follows from Theorem 5

\section{Acknowledgement}

We want to thank an anonymous referee for carefully reading the paper and giving very helpful comments.

\section{References}

[1] A. Brandstädt, V.B. Le, and J. Spinrad, Graph classes: a survey. SIAM Monographs on Discrete Math. Appl. 3, SIAM, Philadelphia, 1999.

[2] E. Camby, O. Schaudt, A Note on Connected Dominating Set in Graphs Without Long Paths And Cycles. Submitted.

[3] J. Cardinal, E. Levy, Connected vertex covers in dense graphs, Theor. Comput. Sci. 411 (2010), pp. 2581-2590.

[4] B. Escoffier, L. Gourvès, J. Monnot, Complexity and Approximation Results for the Connected Vertex Cover Problem in Graphs and Hypergraphs, J. of Discrete Algorithms 8 (2010), pp. 36-49. 
[5] H. Fernau, D. Manlove, Vertex and edge covers with clustering properties: Complexity and algorithms, J. of Discrete Algorithms 7 (2009), pp. 149-167.

[6] J. Guo, R. Niedermeier, S. Wernicke, Parameterized complexity of generalized vertex cover problems, Algorithms and data structures (2005), pp. 35-48.

[7] J. Fulman, A note on the characterization of domination perfect graphs, J. Graph Theory 17 (1993) pp. 47-51.

[8] E. Levy. (2009). Approximation Algorithms for Covering Problems in Dense Graphs. Ph.D. thesis. Université libre de Bruxelles, Brussels.

[9] D. Mölle, S. Richter, P. Rossmanith, Enumerate and expand: Improved algorithms for connected vertex cover and tree cover, Theory of Computing Systems 43 (2008), pp. 234-253.

[10] D. Mölle, S. Richter, P. Rossmanith, Enumerate and expand: New runtime bounds for vertex cover variants, Computing and Combinatorics (2006), pp. 265-273.

[11] D.J. Rose, G.S. Lueker, R.E. Tarjan, Algorithmic aspects of vertex elimination on graphs, SIAM Journal on computing (1976), pp. 266-283.

[12] O. Schaudt, On graphs for which the connected domination number is at most the total domination number, Discrete Appl. Math. 160 (2012), pp. 1281-1284.

[13] H. Spakowski, J. Vogel: $\Theta_{2}^{p}$-Completeness: A Classical Approach for New Results. FSTTCS 2000, pp. 348-360.

[14] I.E. Zverovich, Perfect connected-dominant graphs. Discuss. Math. Graph Theory 23 (2003), pp. 159-162.

[15] I.E. Zverovich, V.E. Zverovich, A semi-induced subgraph characterization of upper domination perfect graphs, J. Graph Theory 31 (1999), pp. 29-49. 
